\title{
Haben Wundinfektionen im Sommer Hochsaison?
}

\author{
In den warmen Sommermonaten steigt in Kliniken offenbar das Risiko für postoperative \\ Wundinfektionen. Forscher aus den USA spekulieren daher, ob es sinnvoll sein könnte, elektive \\ Operationen möglichst in kühlere Monate zu legen.
}

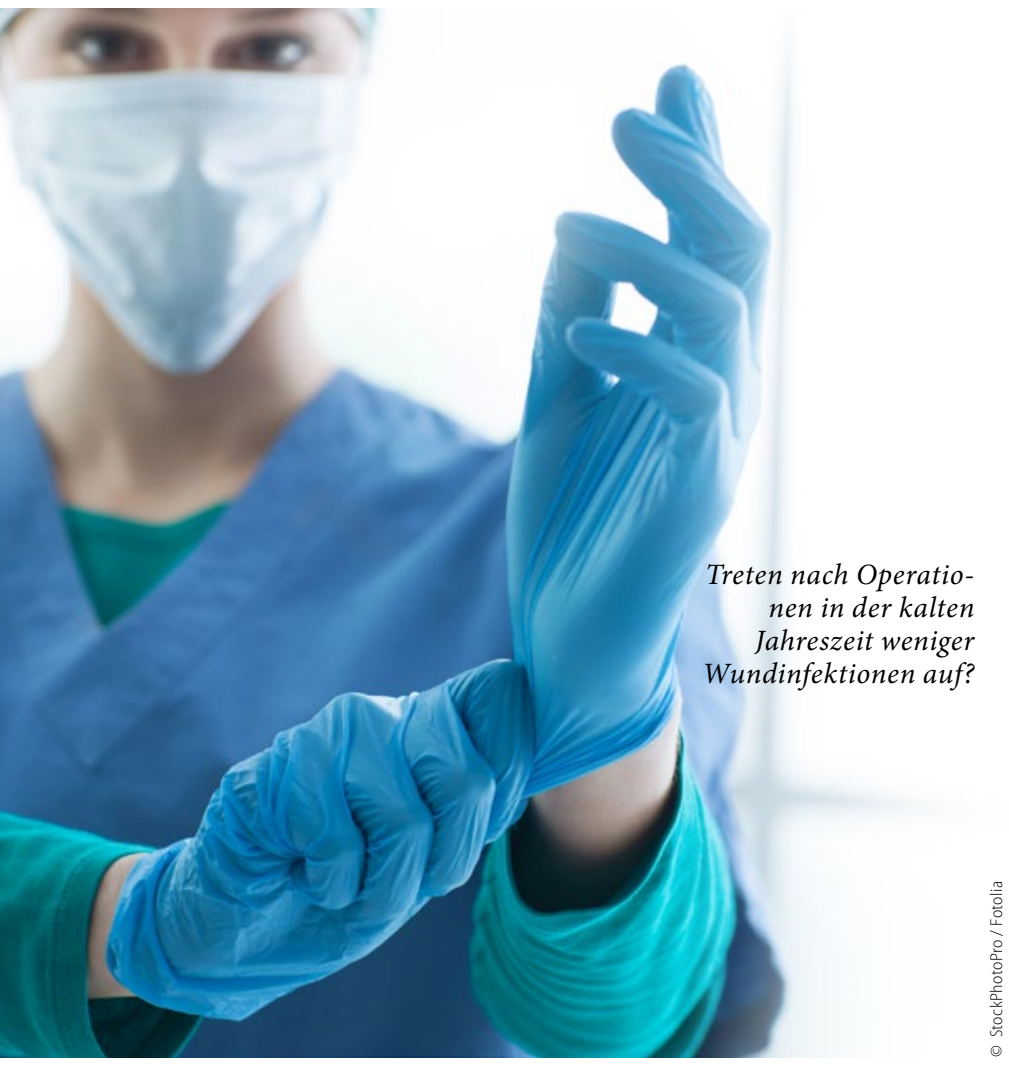

Um herauszufinden, ob die Inzidenz postoperativer Wundinfektionen (SSI, „surgical site infections“) bei hohen Außentemperaturen steigt, hat das Team um Dr. Chris A. Anthony von der Universität Iowa SSI-Fälle aus 4.532 US-Kliniken mit den lokalen Klimadaten zusammengeführt.

In den Jahren zwischen 1998 und 2011 wurden insgesamt 235.969 SSI-Fälle erfasst. Diese wurden 55.429.859 Kontrollpatienten gegenübergestellt. Über diesen Zeitraum hinweg wurde für jeden Monat die Wahrscheinlichkeit einer postoperativen Wundinfektion errechnet. Daraus ergab sich, dass jeweils im Spitzenmonat August die Zahl der Fälle signifikant, nämlich im Mittel um $27 \%$ (2.312 Fälle) gegenüber Januar, dem Monat mit der niedrigsten Inzidenz, angestiegen war.

\section{Deutlich mehr Infektionen im August als im Januar}

In einem besonders warmen August kam es gar zu einem Anstieg um $56 \%$ gegenüber Januar - ein doppelt so starker
Effekt wie zum Beispiel der Effekt durch Diabetes. Laut Anthony et al. hing das Phänomen nicht etwa mit dem sommerlichen Belegschaftswechsel zusammen: Im Juli werden zwar in Lehrkrankenhäusern in den USA die in Ausbildung befindlichen Ärzte ausgetauscht. Allerdings fand sich kein Unterschied zwischen Kliniken, die ausbilden, und solchen, die dies nicht im Programm haben.

Mit dem SSI-Risiko assoziiert war dagegen die monatliche Durchschnittstemperatur: Pro Anstieg um $2,8^{\circ} \mathrm{C}$ nahm die Zahl der Neueinweisungen aufgrund von SSI um 2,1 \% zu. In der Spitzengruppe mit Patienten, die bei Temperaturen über $32^{\circ} \mathrm{C}$ operiert wurden, lag die Wahrscheinlichkeit einer SSI-bedingten Einweisung um $29 \%$ höher als beim Temperaturnadir von weniger als $4,4^{\circ} \mathrm{C}$.

Der Effekt ließ sich in allen geografischen Regionen nachweisen, bei Männern wie bei Frauen und über alle Altersgruppen hinweg. Deutlich am stärksten betroffen waren allerdings Patienten zwischen 40 und 50. Bei ihnen lag die Wahrscheinlichkeit, wegen einer postoperativen Wundinfektion erneut in die Klinik zu kommen, fast dreimal so hoch wie bei 18- bis 30-Jährigen.

\section{Ursache für erhöhtes Infektionsrisiko im Sommer bleibt unklar}

Die Forscher haben nun errechnet, was es bringen würde, wenn man in den heißesten Monaten die Zahl der Operationen um ein Viertel senkt. Das Resultat wäre eine Reduktion der SSI-Fälle um mehr als $20 \%$. „Würden wir einige elektive Patienten statt in den sehr warmen Sommermonaten $\mathrm{zu}$ einem anderen Zeitpunkt operieren, könnten wir sowohl Infektionen reduzieren als auch Kosten sparen“, spekulieren Anthony und seine Koautoren.

Worauf genau das erhöhte Infektionsrisiko im Sommer zurückzuführen ist, bleibt allerdings unklar. Die Wissenschaftler vermuten, dass sich bei höheren Temperaturen möglicherweise mehr bakterielle Erreger auf bestimmten Körperarealen des Patienten ansammeln.

Bevor man entsprechende Empfehlungen abgeben könne, müsse man jedoch erst noch detailliertere Wetterdaten zusammentragen. Insbesondere müsse geklärt werden, ob es Eingriffe gibt, bei denen das SSI-Risiko bei Hitze in die Höhe schießt, und andere, die diesbezüglich vom Wetter weitgehend unabhängig sind.

Dr. Elke Oberhofer 